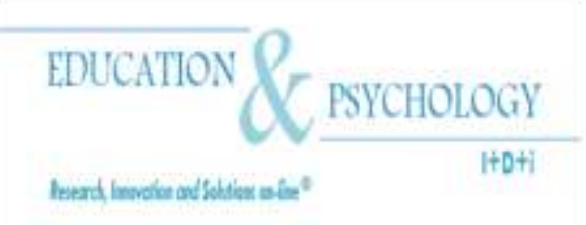

\title{
Análisis comparativo sobre las actitudes de los estudiantes hacia sus compañeros con discapacidad
}

\section{Raquel Suriá Martínez}

Universidad de Alicante. Departamento de Comunicación y Psicología Social. Grupo de Innovación Tecnológica Educativa de Psicología Social (GPSOC), Alicante

\section{España}

Correspondencia: Raquel Suriá Martínez. Departamento de Comunicación y Psicología Social. Edificio de Cienncias Sociales, Despacho 0034PB016, Universidad de Alicante. Carretera San Vicente del Raspeig s/n 03690 San Vicente del Raspeig - Alicante, España. E-mail: raquel.suria@ua.es 


\section{Resumen}

Introducción. Desde el ámbito educativo se ha incrementado el interés hacia la integración del alumnado con discapacidad, siendo las actitudes hacia este colectivo uno de los aspectos de estudio más relevantes. Así, aunque existe en nuestro país una gran cantidad de investigaciones en esta materia, son escasas las centradas en las actitudes de los estudiantes de diferentes etapas educativas. El presente estudio examina las actitudes de los estudiantes hacia sus compañeros con discapacidad en función del curso y etapa formativa. Asimismo queremos comprobar si en las actitudes influye el interaccionar con personas con discapacidad.

Método. Participaron 626 estudiantes. De ellos, 360 pertenecían a la universidad de Alicante y 266 procedían de un instituto de secundaria de esta misma localidad (España). Diseñamos un cuestionario que consta de una escala tipo Likert de 1 al $6(1=$ Totalmente en desacuerdo, $6=$ Totalmente de acuerdo) de 8 ítems donde se estiman las actitudes de los estudiantes. Los datos se analizaron mediante diversos Análisis de Covarianza y univariados.

Resultados. Los resultados indican diferencias estadísticamente significativas en las actitudes de los estudiantes participantes en función del curso, la etapa formativa y el trato con personas discapacitadas.

Discusión y conclusiones. Nuestros resultados sugieren que los estudiantes universitarios indican una actitud más positiva que los alumnos de secundaria. Asimismo, parece que los alumnos que interaccionan con compañeros que padecen alguna discapacidad indican mayor nivel de sensibilización hacia la discapacidad.

Palabras Clave: alumnos con discapacidad; discriminación; actitudes; etapas formativas; interacción

Recepción: 18/10/10 Aceptación Inicial: 20/10/10

Aceptación Definitiva: 23/03/11 


\title{
Comparative analysis of students' attitudes toward their classmates with disabilities
}

\begin{abstract}
Introduction. The interest towards the integration of students with disabilities has increased in the education sector. The attitudes toward this group are one of the most relevant aspects of study. In our country there is a lot of research on this topic, but none of them focused on students at different stages. Our study examines students' attitudes toward students with disabilities depending on their course and formative stage. Also, if we compare the attitude influences meet some students with disabilities.
\end{abstract}

Method. 626 students participated. Of these, 360 of the University of Alicante and 266 from a secondary school in the same locality (España). We designed a questionnaire consisting of a Likert scale of 1 to $6(1=$ Strongly Disagree, $6=$ strongly agree $)$ to 8 items which we estimate the attitudes of students. The data were analyzed using various covariance and univariate analysis.

Results. The results indicate differences in the attitudes of participating students on the basis of the course, the formative stage and the deal with people with disabilities.

Discussion and conclusions. Our results suggest that university students show a more positive attitude than high school students. It also appears that students who interact with disabled peers indicate greater level of awareness of disability.

Keywords: students with disabilities; discrimination; attitudes; interaction; formative stages.

Received: 18/10/10 Initial Acceptance: 10/20/10 Definitive Acceptance: 03/23/11 


\section{Introducción}

En los últimos años, desde la entrada en vigor, en España, de la nueva Ley Orgánica de Modificación de la Ley Orgánica de Universidades (2007), en la que se indica la elaboración de planes destinados a personas con necesidades especiales, el tratamiento que se ha dado a la diversidad en el sistema educativo español ha ido cobrando importancia, atendiéndose cada vez más a las diferencias individuales y a la mejorade nuevos medios de acceso curricular a los alumnos que presentaran discapacidad (Bueno, 2010; Castellana y Sala, 2005; Eches y Ochoa, 2005; Rama, 2005; Suriá, 2006). Een la medida en que ha mejorado la atención educativa que reciben estos estudiantes, se ha facilitado su acceso a la universidad, de manera que el número de estudiantes con discapacidad que ingersan a la educación superior ha ido creciendo de forma notable (Eches y Ochoa, 2005; Granados, 2000; Konur, 2006).

En este sentido, entre las medidas que la mayoría de universidades de este país están integrando debemos resaltar la creación de los Servicios de Atención al alumnado con discapacidad ya que desde estos, se desarrollan una serie de acciones de asesoramiento y apoyo a la comunidad universitaria que contribuyen a suprimir barreras para el acceso, y con ello, al fomento de la participación e inclusión social de los universitarios con discapacidad (Arana, Garcia Meilán y Rodriguez, 2008; Bausela, 2004; Center y Ward, 1987; Clough y Lindsay, 1991).

Paralelamente a la importancia que tienen las adaptaciones y recursos físicos, otro de los aspectos fundamentales para la total integración del alumnado con discapacidad hace referencia a las relaciones interpersonales que establecen los alumnos con discapacidad con el resto de miembros de la comunidad universitaria (Alonso, Navarro y Lidón, 2008; Bueno y Mirambel, 2003; Polo Sánchez y López Justicia, 2005; Verdugo, Arias y Jenaro, 1994).

En relación a esto, la investigación reciente acerca de las variables que favorecen la inclusión, señala que las actitudes son un aspecto fundamental en el éxito de las personas con discapacidad en sus procesos de integración y de éxito en su proceso de enseñanza aprendizaje (Bueno, 2010; Castejón, 2004; Jarvis y French, 1990; Verdugo et al., 1994).

Entre las múltiples definiciones existentes sobre las actitudes, Ovejero (1998), concluye que "la actitud es una predisposición aprendida para responder consistentemente de un 
modo favorable o desfavorable ante objetos, personas o grupos de personas y situaciones" (p. 193). Basándonos en esta definición, según las actitudes que tengamos hacia las personas influirán en la interacción con ellas, de forma que, en función de la concepción que tengamos sobre las personas con discapacidad así será nuestra predisposición a comportarnos en nuestras relaciones con ellas, y con ello, a influir en su integración en la sociedad (Altman, 1981; Granados, 2000).

Asimismo, tal y como indica el mismo autor, las actitudes que tenemos son aprendidas, lo cual se traduce en que se van formando a lo largo de la vida y no en un momento determinado del desarrollo evolutivo de la persona. Por tanto, ¿podríamos pensar que pueden cambiar o evolucionar las actitudes hacia los estudiantes con discapacidad en función de la edad?, o bien, trasladándolo al ámbito educativo, ¿podrían existir diferencias en las actitudes según la etapa formativa que cursen los estudiantes? o incluso, ¿podrían existir diferencias entre los diferentes cursos de la misma etapa educativa?

En este sentido, un estudio llevado a cabo por Infante y Gómez, (2004) examinó las actitudes mostradas por estudiantes universitarios de diversas carreras, concretamente de Construcción Civil, Ingeniería, Magisterio Infantil y Pedagogía. Los resultados indicaron que las actitudes eran más positivas en los estudiantes del último curso. También con fines comparativos, Gughwan y Chow (2001), examinaron las actitudes hacia la diversidad en varios grupos de estudiantes de diferentes etapas. Los resultados indicaron que los estudiantes de posgrado indicaron actitudes más favorables hacia la integración de personas con discapacidad que estudiantes preuniversitarios. Parece, por tanto, que en las actitudes que se tengan hacia las personas con discapacidad van a influir aspectos como la edad, el nivel educativo y la etapa de formación.

A su vez, y basándonos en las cuestiones anteriores, es esperable que, si las actitudes se van adquiriendo a lo largo de la vida en función de las experiencias que se hayan vivido, de algún modo, la convivencia con personas con discapacidad puede influir en la percepción que se tenga de ellas y con ello, en su actitud hacia ellas, así, ¿existen diferencias en las actitudes que los estudiantes tienen de los alumnos discapacitados según tengan o no interacción con ellos?, ¿Qué variables de la persona con discapacidad pueden influir en las actitudes que se tengan hacia ella? Para dar respuesta a esto, algunos autores han enfatizado el papel del tipo y grado de discapacidad de la persona afectada (Chan et al., 1988; Kowalski y Rizzo, 
1996; Yuker y Block, 1986). En este sentido, la predisposición suele ser más adecuada ante estudiantes con discapacidades leves que severas (Rizzo y Vispoel, 1991).

Por tanto, es en este contexto en el que cobra relevancia nuestro estudio puesto que las actitudes son una de las variables más importantes a la hora de facilitar o dificultar el proceso de integración e inclusión de alumnos con discapacidad en el ámbito educativo (Arnaiz, 2003; Konur, 2006; Livneh, 1991; Reina, 2003). De este modo, pensamos que examinando las actitudes hacia los estudiantes con discapacidad que tienen los alumnos procedentes de diferentes etapas, se puede llevar a cabo una intervención para modificarlas o mejorarlas en lo posible.

\section{Objetivos e hipótesis}

Los objetivos del estudio estñan centrados en averiguar si existen diferencias entre las actitudes de los estudiantes hacia los alumnos con discapacidad en función del curso, así como de la etapa formativa en la que se encuentra la muestra de alumnos participantes del estudio. En este sentido, hemos mencionado que las actitudes son aprendidas, y por tanto modificables, de manera que, si partimos de que el sistema educativo actual debe ser integrador, suponemos que a medida que los alumnos avanzan en los diferentes ciclos formativos deben incrementar sus actitudes positivas.

A su vez, si las actitudes se van adquiriendo a lo largo de la vida, en función de las experiencias que se hayan vivido podemos pensar que las actitudes que los estudiantes van adquiriendo a través de las diferentes etapas pueden variar en función de la experciencia de conocer a personas con discapacidad o no, por tanto esperamos:

Hipótesis 1. Existirán, en las etapas formativas superiores, una actitud más favorable hacia las personas con discapacidad que en periodos formativos más tempranos.

Hipótesis 2. Existirán diferencias significativas en las actitudes que los estudiantes tienen hacia los alumnos discapacitados según tengan o no interacción con ellos.

\section{Método}

\section{Participantes}


La muestra fue seleccionada por conveniencia, y la formaron 626 alumnos, procedentes de la universidad de Alicante y del instituto de enseñanza secundaria Figueras Pacheco de la ciudad de Alicante. De ellos, 354 (56.5\%) eran mujeres, 148 (23.6\%) varones y 124 (19.8\%) no identificaron su género. Los alumnos fueron clasificados en distintos grupos en función del curso y de la etapa formativa (Tabla 1).

\section{Tabla 1. Frecuencia y porcentaje de alumnos en función del curso y de la etapa formativa}

\begin{tabular}{lcc}
\hline Nivel de estudios & $\mathrm{N}$ & Porcentaje \\
\hline ESO & & \\
\hline $1^{\circ} \mathrm{ESO}$ & 109 & 17.3 \\
$2^{\circ} \mathrm{ESO}$ & 38 & 6 \\
$3^{\circ} \mathrm{ESO}$ & 47 & 7.4 \\
$4^{\circ} \mathrm{ESO}$ & 66 & 10.5 \\
$\quad$ Total & 266 & 42.5 \\
\hline Universidad & & \\
\hline $1^{\circ}$ Universidad & 124 & 19.7 \\
$2^{\circ}$ Universidad & 126 & 20 \\
$3^{\circ}$ Universidad & 119 & 18.9 \\
$\quad$ Total & 360 & 57.5 \\
Total alumnos & 626 & 100 \\
\hline
\end{tabular}

\section{Instrumentos}

El instrumento empleado ha sido un cuestionario elaborado y validado para el estudio (ver anexo I), el cual está formado por dos bloques. En el primero aparecen los datos referentes a los datos sociodemográficos del alumnado, como son, el sexo, el curso y la etapa de estudio. La segunda parte del instrumento consta de una escala tipo Likert de 1 a 6 (1= Totalmente en desacuerdo, $6=$ Totalmente de acuerdo) de 8 ítems relacionados con las creencias y actitudes de los estudiantes hacia los estudiantes con discapacidad. La consistencia interna del cuestionario se obtuvo a través del coeficiente Alfa de Cronbach, el cual indicó un $84 \%$ de fiabilidad, $(\mathrm{r}=.84)$, por lo que constatamos que esta escala posee una fiabilidad aceptable.

\section{Diseño}

Nuestra investigación se basa en un diseño cuasi-experimental ya que controlamos la variable interacción con personas con discapacidad. En este sentido, pensamos que la convivencia con personas con discapacidad puede influir en la percepción que se tenga de ellas y 
con ello, en su actitud hacia ellas. Por otro lado, no se distribuye la población al azar puesto que los estudiantes pertenecen a contextos concretos y agrupados en función del curso y etapa de formación.

\section{Análisis Estadístico}

Para hallar las comparaciones entre los grupos en los diferentes items de la escala de evaluación, se analizaron utilizando análisis multivariado de covarianza (MANCOVA). Se decidió utilizar una covariante (conocer a una persona con discapacidad) para controlar que las diferencias entre los grupos no se viesen afectadas por esta variable. Sin el control estadístico (mediante la covariante) de dicha variable, los efectos observados podrían explicarse, no por las diferencias entre los grupos en sus actitudes en función del curso y etapa, sino por el efecto de la interacción con personas discapacitadas.

\section{Resultados}

Según los resultados indicados por los 626 alumnos de la muestra observamos (Figura 1) que en todos los ítems las puntuaciones medias se encuentran entre estar "totalmente en desacuerdo", en "bastante en desacuerdo" y en "dudo, pero algo en desacuerdo"

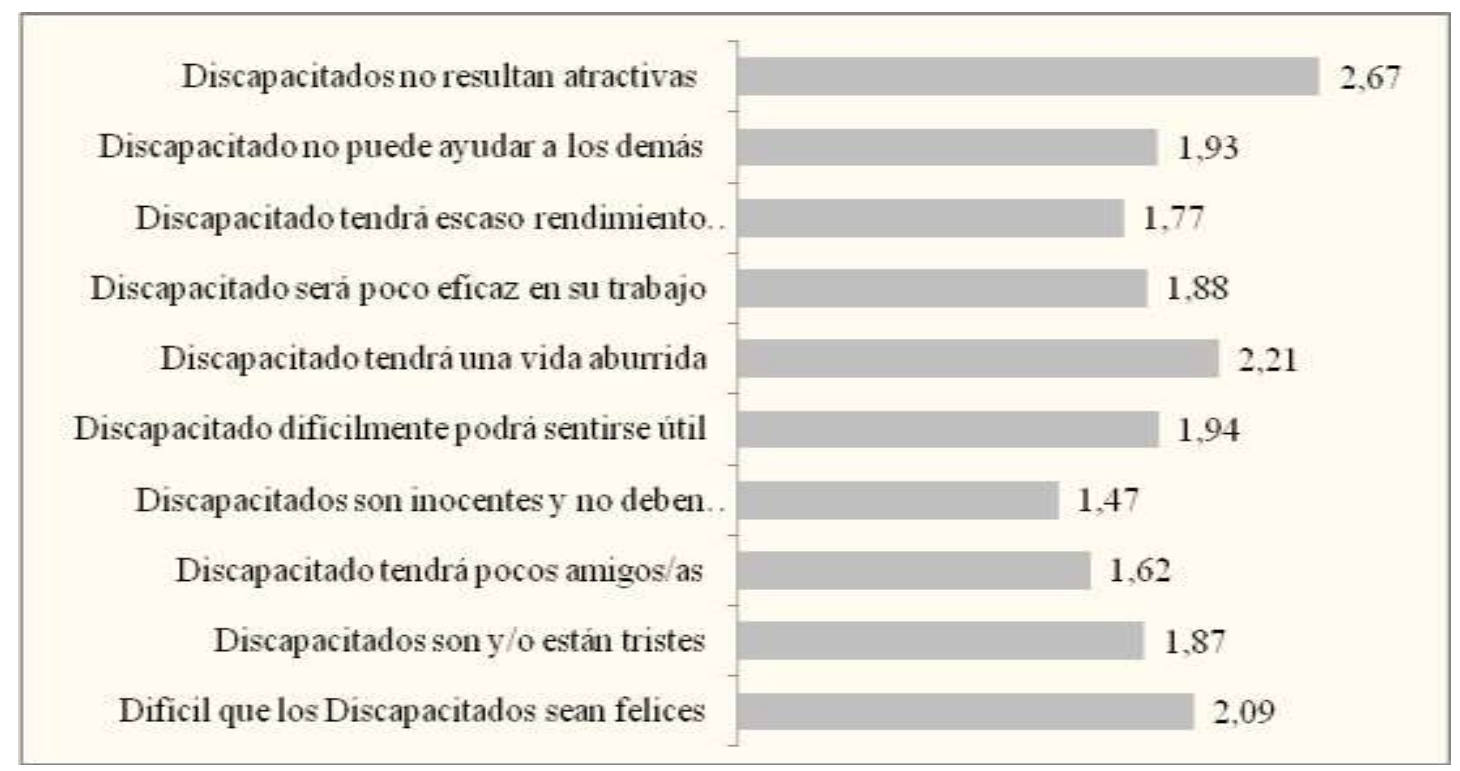


Figura 1. Puntuaciones medias de los estudiantes en la escala

\section{Dimensiones en función de la etapa formativa}

En la Figura 1, se presentan los valores medios obtenidos para cada una de los items de la escala. El MANCOVA general fue significativo [Lambda de Wilks $(10.587)=17.94$, p=.000]. En la Figura 2 se presentan los resultados de los ANCOVAs univariantes.Como puede comprobarse, excepto para los items relativos a la tristeza, a la vida sexual y a resultar atractivos, es el grupo de estudiantes de secundaria el que presenta puntuaciones más altas que los estudiantes universitarios es decir, los que más de acuerdo están con estas afirmaciones. Así, se observa que ante el ítem referente a "Es muy difícil que las personas con discapacidad sean felices" la media de los alumnos que cursan secundaria $(\mathrm{M}=2.58, \mathrm{D} . \mathrm{T}=1.53)$, es más alta a la de los alumnos que cursan estudios universitarios $(\mathrm{M}=1.74, \mathrm{D} . \mathrm{T}=1.29)$.

Con respecto al ítem que hace referencia a que "Una persona con discapacidad tendrá pocos amigos/as", observamos que el grupo de secundaria $(\mathrm{M}=1.99$, D.T.=1.05), tiene una media superior al grupo universitario $(\mathrm{M}=1.35, \mathrm{D} . \mathrm{T}=0.72)$. En la siguiente afirmación relativa a "Una persona con discapacidad difícilmente podrá sentirse útil” observamos que el grupo de la ESO, destacaron con una media de 2.53 (D.T=1.66), y el grupo universitario una media igual a 1.5 (D.T=1.03). Ante la afirmación relativa a creer que "Una persona con discapacidad tendrá una vida aburrida", encontramos que los alumnos de la ESO, alcanzaron una puntuación media de 2.73 (D.T=1.53), mientras que los estudiantes universitarios obtuvieron una media de 1.82 (D.T=1.16). En cuanto al ítem "Una persona con discapacidad será poco eficaz en su trabajo "Una persona con discapacidad difícilmente podrá sentirse útil” observamos que el grupo de la ESO, destacaron con una media superior $(\mathrm{M}=2.28$, D.T=1.34), que el grupo universitario $(\mathrm{M}=1.58$, D.T=1.05). Con respecto a "Una persona con discapacidad tendrá escaso rendimiento escolar" observamos que el grupo de la ESO, destacó con una media de 2.21 (D.T=1.45), y el grupo universitario una media igual a 1.45 (D.T=0.96).

Por último, al examinar el ítem relativo a "Es difícil que una persona con discapacidad pueda ayudar a los demás"observamos que el grupo de la ESO, destacó con una media de $2.44(\mathrm{DT}=1.46)$, y el grupo universitario una media igual a $1.55(\mathrm{DT}=1.01)$. 


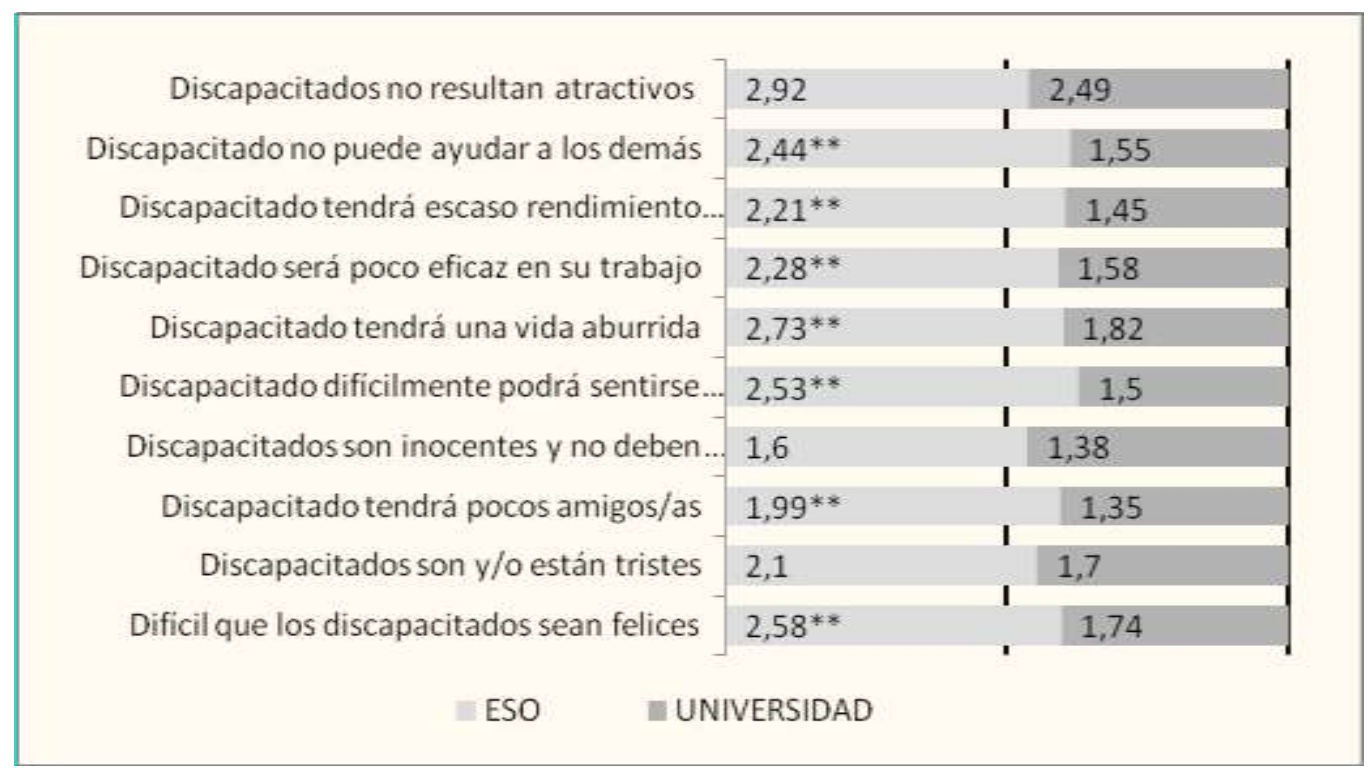

$(* *)=$ Nivel de Significación de .001, (*)= Nivel de Significación de.05

Figura 2. Medias de la escala en función de la etapa formativa

Al examinar si la interacción con las personas con discapacidad influye en las actitudes hacia estos (Tabla 2), los resultados indicaron la influencia de la covariable, observando que los alumnos que piensan en alguien en concreto muestran unas actitudes más positivas en todos los ítems de la escala a un nivel de probabilidad en torno al $1 \%(\mathrm{p} \leq .001)$.

Tabla 2. ANCOVAs según etapa formativa e interacción con personas con discapacidad

\begin{tabular}{llll}
\hline Variable & Dimensiones & F & Significación \\
\hline Persona conocida & & & \\
\hline & Feliz & 52.82 & .000 \\
& Tristeza & 228.1 & .000 \\
& Amigos & 6.19 & .013 \\
& Sexualidad & 11.49 & .001 \\
& Util & 126.97 & .000 \\
& Aburrida & 173.47 & .000 \\
& Trabajo & 58.70 & .000 \\
& Rendir & 71.95 & .000 \\
& Ayudar & 44.39 & .000 \\
& Atractiva & 88.45 & .000 \\
\hline Etapa formativa & & & \\
\hline & Feliz & 38.4 & .000 \\
& Tristeza & 0.215 & .643 \\
& Amigos & 60.97 & .000 \\
& Sexualidad & 3.724 & .054 \\
& Util & 63.93 & .000 \\
& Aburrida & 36.28 & .000 \\
& Trabajo & 41.26 & .000 \\
& Rendir & 35.01 & .000
\end{tabular}




\begin{tabular}{lll} 
Ayudar & 49.03 & .000 \\
Atractiva & 2.55 & .110 \\
\hline
\end{tabular}

\section{Dimensiones en función del curso}

Se llevó a cabo un segundo MANCOVA para comparar las puntuaciones de la escala entre los estudiantes en función del curso en el que estabn matriculados los estudiantes. Al igual que con la etapa formativa, la variable sobre si conocen o interaccionan con alguien con discapacidad fue la covariante. El MANCOVA general fue significativo [Lambda de Wilks $(10.582)=39.72, \mathrm{p}=.000]$.

En la Tabla 3 se presentan los resultados de los ANCOVAs univariantes y los análisis post hoc de las puntuaciones de las escalas. De esta forma, podemos observar que, al examinar las puntuaciones medias de las dimensiones de la escala en función del curso en el que se encuentran los alumnos encontramos diferencias estadisticamente significativas en todos los ítems.

Tabla 3. ANOVAs y descriptivos según curso

\begin{tabular}{|c|c|c|c|c|c|c|c|c|c|}
\hline \multirow[t]{2}{*}{ Dimensiones } & \multicolumn{9}{|c|}{ Curso } \\
\hline & D. $T$ & $\begin{array}{l}1^{\circ} \\
\text { ESO }\end{array}$ & $\begin{array}{l}2^{\circ} \\
\text { ESO }\end{array}$ & $\begin{array}{ll}3^{\circ} \\
\text { ESO }\end{array}$ & $\begin{array}{l}4^{\circ} \\
\text { ESO } \\
\end{array}$ & $\begin{array}{l}1^{\circ} \\
\mathrm{UNI}\end{array}$ & $\begin{array}{l}2^{\circ} \\
\mathrm{UNI}\end{array}$ & $\begin{array}{l}3^{\circ} \\
\mathrm{UNI}\end{array}$ & $\mathrm{F}$ \\
\hline $\begin{array}{l}\text { Difícil que los disca- } \\
\text { pacitados sean felices }\end{array}$ & $\begin{array}{l}\text { M } \\
\text { D.T }\end{array}$ & $\begin{array}{l}2.73_{\text {bg }} \\
1.49\end{array}$ & $\begin{array}{l}2.47_{\mathrm{ch}} \\
1.62\end{array}$ & $\begin{array}{l}2.21 \\
1.10\end{array}$ & $\begin{array}{l}2.71_{\mathrm{df}} \\
1.73\end{array}$ & $\begin{array}{l}1.42_{\mathrm{abcd}} \\
0.82\end{array}$ & $\begin{array}{l}1.51_{\text {efgh }} \\
1.18\end{array}$ & $\begin{array}{l}2.29_{\mathrm{ae}} \\
1.60\end{array}$ & $16.3 * *$ \\
\hline $\begin{array}{l}\text { Discapacitados son y/o } \\
\text { están tristes }\end{array}$ & $\begin{array}{l}\text { M } \\
\text { D.T }\end{array}$ & $\begin{array}{l}2.11 \\
0.77\end{array}$ & $\begin{array}{l}2.57_{\mathrm{ab}} \\
0.47\end{array}$ & $\begin{array}{l}1.98 \\
0.94\end{array}$ & $\begin{array}{l}2.42_{\mathrm{c}} \\
1.12\end{array}$ & $\begin{array}{l}1.35_{\mathrm{a}} \\
0.65\end{array}$ & $\begin{array}{l}1.20_{\mathrm{bc}} \\
0.70\end{array}$ & $\begin{array}{l}1.68 \\
1.12\end{array}$ & $6.8 * *$ \\
\hline $\begin{array}{l}\text { Discapacitado tendrá } \\
\text { pocos amigos/as }\end{array}$ & $\begin{array}{l}\text { M } \\
\text { D.T }\end{array}$ & $\begin{array}{l}1.84_{\text {bgi }} \\
0.84\end{array}$ & $\begin{array}{l}1.39_{\mathrm{j}} \\
1.08\end{array}$ & $\begin{array}{l}1.91_{\mathrm{cek}} \\
1.14\end{array}$ & $\begin{array}{l}2.67_{\text {athijk }} \\
0.98\end{array}$ & $\begin{array}{l}1.37_{\mathrm{abc}} \\
0.73\end{array}$ & $\begin{array}{l}1.12_{\text {defg }} \\
0.47\end{array}$ & $\begin{array}{l}1.57_{\mathrm{dh}} \\
0.86\end{array}$ & $30.3 * *$ \\
\hline $\begin{array}{l}\text { Discapacitados no } \\
\text { deben tener actividad } \\
\text { sexual }\end{array}$ & $\begin{array}{l}\text { M } \\
\text { D.T }\end{array}$ & $\begin{array}{l}1.71_{\mathrm{a}} \\
0.80\end{array}$ & $\begin{array}{l}1.29 \\
0.69\end{array}$ & $\begin{array}{l}1.57 \\
0.80\end{array}$ & $\begin{array}{l}1.64 \\
1.10\end{array}$ & $\begin{array}{l}1.23_{\mathrm{a}} \\
0.73\end{array}$ & $\begin{array}{l}1.33 \\
0.77\end{array}$ & $\begin{array}{l}1.57 \\
0.97\end{array}$ & $4.81 * *$ \\
\hline $\begin{array}{l}\text { Discapacitado difícil- } \\
\text { mente podrá sentirse } \\
\text { útil }\end{array}$ & $\begin{array}{l}\text { M } \\
\text { D.T }\end{array}$ & $\begin{array}{l}3.30_{\text {beghi }} \\
1.79\end{array}$ & $\begin{array}{l}1.55_{\mathrm{i}} \\
1.13\end{array}$ & $\begin{array}{l}2.21_{\mathrm{cf}} \\
1.20\end{array}$ & $\begin{array}{l}2.20_{\text {adh }} \\
1.54\end{array}$ & $\begin{array}{l}1.43_{\mathrm{abc}} \\
0.82\end{array}$ & $\begin{array}{l}1.25_{\text {def }} \\
0.72\end{array}$ & $\begin{array}{l}1.82_{\mathrm{g}} \\
1.37\end{array}$ & $32.4^{* *}$ \\
\hline $\begin{array}{l}\text { Discapacitado tendrá } \\
\text { una vida aburrida }\end{array}$ & $\begin{array}{l}\text { M } \\
\text { D.T }\end{array}$ & $\begin{array}{l}3.75_{\text {chik }} \\
1.42\end{array}$ & $\begin{array}{l}1.42_{\mathrm{jl}} \\
0.83\end{array}$ & $\begin{array}{l}1.91 \\
0.93\end{array}$ & $\begin{array}{l}2.50_{\mathrm{bgkl}} \\
1.34\end{array}$ & $\begin{array}{l}1.73_{\mathrm{abc}} \\
1.04\end{array}$ & $\begin{array}{l}1.40_{\text {fgh }} \\
0.59\end{array}$ & $\begin{array}{l}2.35_{\text {afij }} \\
1.47\end{array}$ & $50.4 * *$ \\
\hline $\begin{array}{l}\text { Discapacitado será } \\
\text { poco eficaz en su tra- } \\
\text { bajo }\end{array}$ & $\begin{array}{l}\text { M } \\
\text { D.T }\end{array}$ & $\begin{array}{l}2.89_{\text {befhi }} \\
1.43\end{array}$ & $\begin{array}{l}1.32_{\mathrm{gh}} \\
0.81\end{array}$ & $\begin{array}{l}1.72_{\mathrm{i}} \\
0.88\end{array}$ & $\begin{array}{l}2.35_{\mathrm{adg}} \\
1.25\end{array}$ & $\begin{array}{l}1.57_{\mathrm{ab}} \\
0.99\end{array}$ & $\begin{array}{l}1.21_{\text {cde }} \\
0.65\end{array}$ & $\begin{array}{l}1.99_{\mathrm{cf}} \\
1.29\end{array}$ & $28.8^{* *}$ \\
\hline $\begin{array}{l}\text { Discapacitado tendrá } \\
\text { escaso rendimiento }\end{array}$ & $\begin{array}{l}\text { M } \\
\text { D.T }\end{array}$ & $\begin{array}{l}3.19_{\text {acdefg }} \\
1.49\end{array}$ & $\begin{array}{l}1.32_{\mathrm{f}} \\
0.90\end{array}$ & $\begin{array}{l}1.57 \mathrm{~g} \\
1.02\end{array}$ & $\begin{array}{l}1.67_{\mathrm{e}} \\
0.95\end{array}$ & $\begin{array}{l}1.38_{\mathrm{a}} \\
0.78\end{array}$ & $\begin{array}{l}1.17_{b c} \\
0.57\end{array}$ & $\begin{array}{l}1.82_{\mathrm{bd}} \\
1.27\end{array}$ & $44.5^{* *}$ \\
\hline
\end{tabular}


escolar

$\begin{array}{llllllllll}\text { Discapacitado no pue- } & \mathrm{M} & 3.44_{\text {befghi }} & 1.29_{\mathrm{h}} & 1.94_{\mathrm{i}} & 1.91_{\mathrm{dg}} & 1.41_{\mathrm{ab}} & 1.33_{\text {cde }} & 1.92_{\mathrm{acf}} & 50.1^{* *} \\ \text { de ayudar a los demás } & \text { D.T } & 1.49 & 0.46 & 0.96 & 1.05 & 0.71 & 0.90 & 1.24 & \\ \text { Discapacitados no } & \mathrm{M} & 3.39_{\text {cfhi }} & 2.98_{\mathrm{adg}} & 2.26_{\mathrm{i}} & 3.21_{\mathrm{be}} & 2.31_{\mathrm{abc}} & 2.20_{\text {def }} & 1.87_{\mathrm{gh}} & 12.7^{* *} \\ \text { resultan atractivos } & \text { D.T } & 1.54 & 1.53 & 1.26 & 1.59 & 1.41 & 1.38 & 1.46 & \end{array}$

(aa, bb...) Pares de letras iguales indican diferencias estadísticamente significativas entre sus medias; $(* *)=$ Nivel de Significación de .001; $\left(^{*}\right)=$ Nivel de Significación de .05

Así, a nivel descriptivo, observamos que en todas las dimensiones de la escala encontramos que los estudiantes de cursos pertenecientes a la ESO indican unos índices centrales más elevados que los alumnos universitarios, siendo interesante resaltar que en la mayoría de ítems destacan los estudiantes que se encuentran cursando $1^{\circ}$ de la ESO por tener unas medias más elevadas que los estudiantes pertenecientes al resto de cursos.

Por ejemplo, ante el primer ítem que hace referencia a "Es muy difícil que las personas con discapacidad sean felices" observamos que la media de los alumnos que cursan $1^{\circ}$ de la ESO (M=2.73, D.T=1.49) superan con medias más altas a los alumnos que cursan niveles superiores, encontrándose el grupo de alumnos que cursan $1^{\circ}$ de alguna titulación en la univeridad muestran un nivel medio más bajo $(\mathrm{M}=1.742$, $\mathrm{D} . \mathrm{T}=0.82)$.

Del mismo modo, en la afirmación relativa a "Las personas con discapacidad son inocentes y no deben tener actividad sexual" observamos la misma dirección, es decir, es el grupo de $1^{\circ}$ de la ESO el que destaca con una media más elevada que el resto de los cursos analizados $(M=1.71, D . T=0.8)$. Así, al examinar la media que indica estar más en contra de esta afirmación observamos que vuelve a ser el alumnado de $1^{\circ}$ de la universidad $(\mathrm{M}=1.23$, D.T $=0.73)$.

Al centrarnos en el ítem que hace referencia a que "Una persona con discapacidad difícilmente podrá sentirse útil” observamos que los alumnos de $1^{\circ}$ de la ESO $(\mathrm{M}=3.3$, D.T=1.79), vuelven a destacar con una media superior al resto de grupos. Mients que si examiamos que grupo indica un índice central más inferior, observamos que es el alumnado universitario que pertenece a $2^{\circ}$ curso, $(M=1.25$, D.T $=0.72)$.

Ante la afirmación relativa a creer que "Una persona con discapacidad tendrá una vida aburrida", encontramos que los alumnos que destacan por tener puntuaciones medias más altas siguen siendo los alumnos de $1^{\circ}$ de la ESO $(M=3.75$, D.T=1.42). A su vez, los estudian- 
tes universitarios, concretamente los alumnos de $2^{\circ}$ muestran una media inferior $(M=1.40$, D.T $=0.59)$.

En cuanto al ítem "Una persona con discapacidad será poco eficaz en su trabajo" observamos la misma línea, es decir, los estudiantes del curso de $1^{\circ}$ de la ESO, resaltan con una media superior ( $\mathrm{M}=2.89$, D.T=1.43). De igual modo, es el grupo de alumnos de $2^{\circ}$ de la universidad el que indica una media inferior al resto de grupos $(\mathrm{M}=1.21, \mathrm{D} . \mathrm{T}=0.65)$.

$\mathrm{Si}$ analizamos las medias de los grupos en cuanto al siguiente ítem, el relacionado con la afirmación realativa a "Una persona con discapacidad tendrá escaso rendimiento escolar" observamos que de nuevo, es el curso de $1^{\circ}(\mathrm{M}=3.19$, D.T=1.49) de la ESO el que muestra un íncice central mayor que el del resto de grupos. Asimismo, los estudiantes universitarios que cursan $2^{\circ}$ curso vuelven a ser los que muestran una media inferior $(\mathrm{M}=1.17, \mathrm{D} . \mathrm{T}=0.57)$.

En referencia a creer que "Es difícil que una persona discapacitada pueda ayudar a los demás" observamos puntuaciones medias más altas en $1^{\circ}(\mathrm{M}=3.44$, D.T=1.49) de la ESO, en comparación con el resto de los cursos. De nuevo, si examiamos que grupo indica un índice central más inferior observamos que es el alumnado universitario que cursa $2^{\circ},(M=1.33$, D.T $=0.0 .90)$.

El análisis de los grupos con respecto a la afirmación referente a que "Las personas discapacitadas no resultan atractivas para otras personas" muestra, que los cursos de la ESO destacan con medias más altas que el alumnado universitario, repitiéndose la dirección de los anterioees ítems, en otros términos, destaca $1^{\circ}$ de la ESO $(\mathrm{M}=3.39$, D.T=1.54), mientras que el alumnado de $3^{\circ}$ de la universidad es el que más en contra está de dicha firmación $(M=1.87$, D.T=1.46).

Por su parte, al examinar las medias de los grupos en algunas afirmaciones en las que observamos que no destaca el grupo de $1^{\mathrm{o}}$ de secundaria encontramos que, en la dimensión relativa a creer que "Normalmente las personas con discapacidad son y/o están tristes", destaca el grupo de alumnos de $2^{\circ}$ de la ESO $(M=2.57$, D.T=0.47) por ser más elevada que la del resto de alumnos que cursan los otros cursos, así como la afirmación que hace referencia a "Una persona con discapacidad tendrá pocos amigos/as", observando que los estudiantes que cursan $3^{\circ}$ de la ESO $(\mathrm{M}=1.84$, D.T=0.84), tienen una media superior al resto de los grupos. 
Al examinar si la interacción con las personas con discapaidad influye en las actitudes hacia estos, observamos resultados significativos en todas las dimensiones en función de la covariante (Tabla 4).

Tabla 4. ANCOVAs según curso e interacción con personas con discapacidad

\begin{tabular}{llll}
\hline Variable & Dimensiones & F & Significación \\
\hline Persona conocida & & & \\
\hline & Feliz & 43.33 & .000 \\
& Tristeza & 235.9 & .000 \\
& Amigos & 8.71 & .003 \\
& Sexualidad & 6.0 & .009 \\
& Util & 81.91 & .000 \\
& Aburrida & 108.71 & .000 \\
Trabajo & 26.19 & .000 \\
& Rendir & 27.11 & .000 \\
& Ayudar & 11.15 & .001 \\
& Atractiva & 66.88 & .000 \\
\hline
\end{tabular}




\begin{tabular}{llll}
\hline Curso & & & \\
\hline & Feliz & 10.87 & .000 \\
Tristeza & 6.74 & .000 \\
Amigos & 26.43 & .000 \\
Sexualidad & 2.7 & .013 \\
Util & 19.37 & .000 \\
Aburrida & 27.78 & .000 \\
Trabajo & 22.58 & .000 \\
Rendir & 28.54 & .000 \\
Ayudar & 32.48 & .000 \\
Atractiva & 6.95 & .000 \\
\hline
\end{tabular}

Finalmente, para examinar en que dirección se observa el efecto de la covariable "interacción con personas con discapacidad" observamos (Figura 3), que los alumnos que piensan en alguien en concreto indican unas actitudes más positivas en todos los ítems de la escala a un nivel de probabilidad entre el $1 \%$ y el $5 \%$.

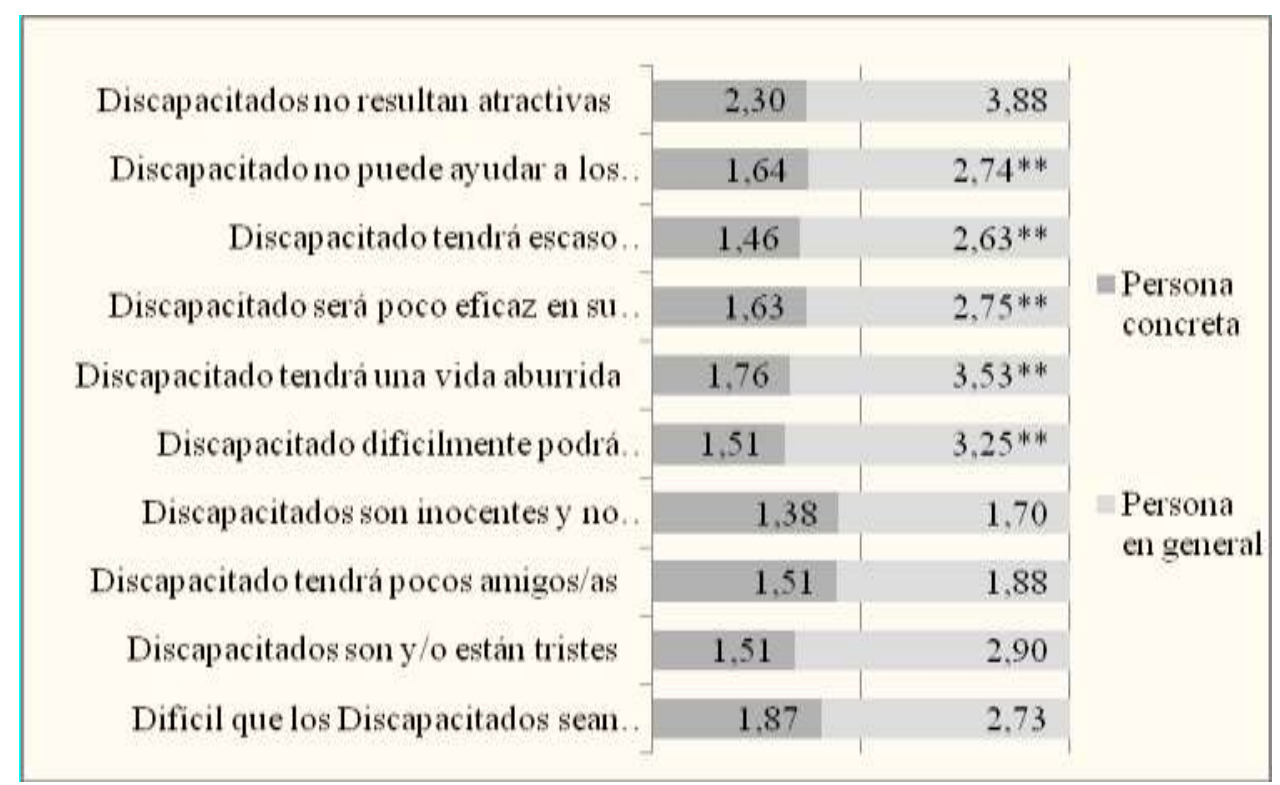

$(* *)=$ Nivel de Significación de $.001,(*)=$ Nivel de Significación de.05

Figura 3. Medias de la escala en función de la interacción con una persona con discapacidad

\section{Discusión y conclusiones}

Los resultados anteriormente expuestos nos permiten llegar a una primera conclusión: se observa que los alumos muestran unas actitudes favorables hacia los alumnos con discapacidad ya que ninguna de las puntuaciones medias analizadas indica estar de acuerdo con actitudes desfavorables hacia los estudiantes con discapacidad. Así, entre los resultados podemos ver que las manifestaciones con las que más se suele estar "totalmente en desacuerdo" son las 
que afirrman que "Las personas con discapacidad son inocentes y no deben tener actividad sexual", "que tendrán pocos amigos" y "que tendrán escaso rendimiento". Por el contrario, las afirmaciones con las que están más de acuerdo y dudo"son las siguientes: "Las personas con discapacidad no resultan atractivas para otras personas" así como, la creencia de que "Una persona con discapacidad tendrá una vida aburrida"

$\mathrm{Al}$ analizar cada ítem de la escala en función de la etapa formativa y del curso, encontramos diferencias estadísticas significativas en las actitudes de los estudiantes. Así, al referirnos a la etapa formativa, observamos que en los ciclos de formación donde los alumnos son más jóvenes muestran actitudes menos favorables que en los ciclos superiores, es decir, parece que los estudiantes de más edad tienden a tener actitudes más positivas hacia la discapacidad. Estos resultados siguen la misma dirección de otras investigaciones con estudiantes universitarios (Alonso, Navarro y Lidón, 2008; Infante y Gómez, 2004), encontrando que los estudiantes de los últimos cursos presentan actitudes más positivas hacia la diversidad.

Por tanto, podríamos pensar en que la evolución de las actitudes guardan relación con la variable edad, sin embargo, al examinar las dimensiones de la escala en función del curso, observamos que nuestros resultados solo refuerzan parcialmente los obtenidos en la hipótesis concerniente a las diferencias en función de la etapa formativa, ya que vemos que los cursos pertenecientes a la ESO, no guardan una relación directamente positiva en función del curso que se estudie, así, encontramos que los alumnos del curso $4^{\circ}$ de la ESO destacan con actitudes menos favorables hacia los discapacitados en algunas dimensiones que los alumnos de $1^{\circ}$ o $2^{\circ}$ de esta misma etapa formativa. Al igual que sugiere Livneh (1991), podríamos pensar en una evolución hacia una actitud más favorable conforme se avanza entre las diferentes etapas, más que dentro de una misma etapa (inter-etapas más que intra-etapas).

En este sentido, otros autores como Gughwan y Chow (2001), se han basado en esta idea para dar explicación al hallar en sus resultados que los estudiantes de posgrado indicaban unas actitudes más favorables hacia las personas con discapacidad que los estudiantes de pregrado.

Finalmente, un dato a tener en cuenta es el resultado obtenido al examinar las actitudes ante las personas con discapacidad en función de si conocen o mantienen contacto con alguna persona discapacitada o no, de forma que, obervamos que los estudiantes encuestados que 
tienen la experiencia de convivencia directa con personas con alguna discapacidad mantienen una actitud ante ellas más positiva que los que no la tienen. Esto sugiere que una manera de propiciar actitudes positivas ante las personas con discapacidad es facilitar el contacto directo y la interacción con ellas.

\section{Limitaciones y prospectivas}

Aunque debemos ser optimistas con los resultados del estudio, en cuanto a las actitudes positivas de los estudiantes hacia los alumnos con discapacidad, debemos ser conscientes de la provisionalidad de las conclusiones, del tamaño de la muestra, ya que que no hubo una estimación de cálculo de tamaño de muestra, puesto que el muestreo no fue probabilístico, sino por conveniencia, así como el ámbito de estudio ya que nos referimos a un único centro de secundaria y a determinadas titulaciones de la universidad de Alicante (España). Igualmente, debemos ser cautos y considerar algunas limitaciones de este estudio relacionadas con el instrumento para recoger la información y con la deseabilidad social. Así, nuestros participantes al realizar un autoinforme sobre las cuestiones referidas en el cuestionario pueden estar sesgando sus respuestas.

Por ello, sería interesante en futuras investigaciones prolongar el período de seguimiento de estudio para evaluar a los participantes una vez transcurrido cierto tiempo, y de este modo, comprobar si existen variaciones en sus respuestas. A su vez, se podrían examinar otras posibles variables en las actitudes de los estudiantes universitarios como puede ser el tipo de discapacidad, así como el grado en el que se manifiesta, ya que éstas pueden afectar también a las actitudes hacia ellos (Chan et al., 1988; Kowalski y Rizzo, 1996; Yuker y Block, 1986). Por último, además de la aplicación de los autoinformes utilizados para conocer lo que opinan los participantes sería interesante para estudios posteriores incluir otros modelos de evaluación como los tests conductuales, con la finalidad de recabar información sobre las actuaciones de los participantes ante estudiantes con discapacidad. 


\section{Referencias}

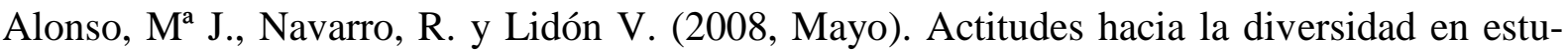
diantes universitarios. Comunicación presentada en las XIII Jornadas de Fomento de la Investigación, Universitat Jaume I, Castellón.

Altman, B. M. (1981). Studies of attitudes toward the handicapped: The need for a new direction. Social Problems, 28, 321-337.

Arana, Martínez J. M., García Meilán, J.J. y Rodríguez, S. (2008). Los Servicios de Atención Psicológica en la universidad española: Análisis crítico. Revista de Psicologia General y Aplicada, 61, 265-284.

Arnaiz, P. (2003). Educación Inclusiva: Una escuela para todos. Málaga: Editorial Aljibe.

Bausela, E. (2004). La función orientadora en el marco de la Universidad. EduPsykhé, 3, 109120.

Bueno, A. (2010). Una mirada ilusionada al futuro de los Servicios de Apoyo a la Discapacidad en la Universidad. Buenas prácticas de apoyo a la discapacidad en la universidad. En A. Bueno, Buenas prácticas durante los estudios universitarios en la recepción de la información (pp. 10-33). Alicante: Editorial CEE Limencop.

Bueno, A. y Mirambel, E. (2003). Escala de Actitudes hacia la Discapacidad en la Universidad: Presentación. Encuentros en Psicología Social 1, 36-39.

Castejón, L. (2004). Percepciones y actitudes sobre el alumno tartamudo en Educación Primaria. Tesis Doctoral. Universidad de Oviedo.

Castellana, M. y Sala, I. (2005, Noviembre). Estudiantes con discapacidad en las aulas universitarias: Estudio sobre la atención a la diversidad dentro de las aulas universitarias. Trabajo presentado en el I Congreso sobre Universidad y Discapacidad. Salamanca, España.

Center, Y. y Ward, J. (1987). Teachers' attitudes towards the integration of disabled children into regular schools. Exceptional Child, 34, 41-56.

Chan, F., Hedl, J.J., Parker, H.J., Lam, C.S., Chan, T. y Yu, B. (1988). Differential attitudes of Chinese students toward people with disabilities: a cross-cultural perspective. The International Journal of Social Psychiatry, 34, 267-273.

Clough, P. y Lindsay, G. (1991). Integrationand the Support Service. Slough: NFER.

Eches, S. y Ochoa, T. (2005). Students with disabilities: Transitioning from High School to higher education. American Secondary Education, 33, 6-20.

Granados, A. (2000). ¿Tiene cabida la diversidad en la Universidad? En, V. Salmerón y V.L. López, Orientación Educativa en las Universidades (pp.144-147). Granada: Grupo Editorial Universitario.

Gughwan, C.H. y Chow, L. (2001). Korean students' differential attitudes toward people with disabilities: an acculturation perspective. International Journal of Rehabilitation Research, 24, 79-81. 
Infante, M. y Gómez, V. (2004). Actitudes de los estudiantes de educación hacia la integración de personas con discapacidad y hacia la educación multicultural. Cultura y Educación, 16, 371-384.

Jarvis, K.C. y French, R. (1990). Attitudes of physical educators toward the integration of handicapped students. Perceptual and Motor Skills, 70, 899-902.

Kowalski, E.M. y Rizzo, T.L. (1996). Factors influencing preservice student attitudes toward individuals with disabilities. Adapted Physical Activity Quarterly, 13, 180-196.

Konur, O. (2006). Teaching disabled students in higher education. Teaching in Higher Education, 11, 351- 363.

Ley Orgánica 4/2007, de 12 de abril, por la cual se modifica la Ley Orgánica 6/2001, de 21 de diciembre, de universidades. (BOE núm. 89, 13 de abril de 2007).

Livneh, H. (1991). On the origins of negative attitudes toward people with disabilities. En R.P. Marinelli y A.E. Dell Orto (Eds), The Psychological and Social Impact of Disability (pp. 181-196). New York: Springer Publishing Company.

Ovejero, A. (1998). Las Relaciones Humanas. Psicología social teórica y aplicada. Madrid: Biblioteca Nueva.

Polo, M. T. y López-Justicia, M. D. (2005). Barreras de acceso al medio físico de los estudiantes con discapacidad motora de la Universidad de Granada. Electronic Journal of Research in Educational Psychology, 3, 121-132.

Rama, C. (2005). Las personas con discapacidades: los excluidos de la educación superior. Trabajo presentado en el seminario "Integración de las personas con discapacidad en la educación superior". República Dominicana, 6 de mayo. Sitio en Internet. Obtenido el 20 de febrero del 2010. En:

http://www.claudiorama.name/archivos/publicaciones/6e3252166468bf1cff451d4dc3d bb441.pdf

Reina, R. (2003). Propuesta de intervención para la mejora de actitudes hacia personas con discapacidad a través de actividades deportivas y recreativas. Obtenido el $10 \mathrm{de}$ febrero del 2010. En: http://www.efdeportes.com/efd59/discap.htm

Rizzo, T.L. y Vispoel, W.P. (1991). Physical educators' attributes and attitudes toward teaching students with handicaps. Adapted Physical Activity Quarterly, 8, 4-11.

Suriá, R. (2006). Docencia, diversidad y discapacidad en el alumnado. Electronic Journal of Research in Educational Psychology, 4, 35-46.

Verdugo, M. A., Arias, B. y Jenaro, C. (1994). Actitudes hacia las personas con minusvalía. Madrid: INSERSO.

Yuker, H. E. y Block, J. R. (1986). Research with the Attitudes Toward Persons Scale (ATDP) 1960-1985. Hempstead, NY: Center for the study of Attitude Toward Persons with Disabilities, Hofstra University. 


\section{Anexo I: Cuestionario}

Indica tu grado de acuerdo o desacuerdo con cada una de las afirmaciones siguientes, según esta numeración:

- Totalmente de acuerdo: ( 6 )

- Bastante de acuerdo: ( 5 )

- Algo de acuerdo, pero dudo: ( 4 )

- Dudo, pero más bien en desacuerdo: ( 3 )

- Bastante en desacuerdo: ( 2 )

- Totalmente en desacuerdo: ( 1 )

\begin{tabular}{|c|c|c|c|c|c|c|}
\hline Escala & $\begin{array}{l}\text { Totalmen- } \\
\text { te de } \\
\text { acuerdo }\end{array}$ & $\begin{array}{l}\text { Bastante } \\
\text { de acuer- } \\
\text { do }\end{array}$ & $\begin{array}{l}\text { Algo de } \\
\text { acuerdo, } \\
\text { pero dudo }\end{array}$ & $\begin{array}{l}\text { Dudo, } \\
\text { pero más } \\
\text { bien en } \\
\text { desacuer- } \\
\text { do }\end{array}$ & $\begin{array}{l}\text { Bastante } \\
\text { en des- } \\
\text { acuerdo }\end{array}$ & $\begin{array}{l}\text { Totalmente } \\
\text { en des- } \\
\text { acuerdo }\end{array}$ \\
\hline $\begin{array}{l}\text { Es muy difícil que las personas con } \\
\text { discapacidad sean felices }\end{array}$ & & & & & & \\
\hline $\begin{array}{l}\text { Normalmente las personas con } \\
\text { discapacidad son y/o están tristes }\end{array}$ & & & & & & \\
\hline $\begin{array}{l}\text { Una persona con discapacidad } \\
\text { tendrá pocos amigos/as }\end{array}$ & & & & & & \\
\hline $\begin{array}{l}\text { Las personas con discapacidad son } \\
\text { inocentes y no deben tener activi- } \\
\text { dad sexual }\end{array}$ & & & & & & \\
\hline $\begin{array}{l}\text { Una persona con discapacidad } \\
\text { difícilmente podrá sentirse útil }\end{array}$ & & & & & & \\
\hline $\begin{array}{l}\text { Una persona con discapacidad } \\
\text { tendrá una vida aburrida }\end{array}$ & & & & & & \\
\hline $\begin{array}{l}\text { Una persona con discapacidad será } \\
\text { poco eficaz en su trabajo }\end{array}$ & & & & & & \\
\hline $\begin{array}{l}\text { Una persona con discapacidad } \\
\text { tendrá escaso rendimiento escolar }\end{array}$ & & & & & & \\
\hline $\begin{array}{l}\text { Es difícil que una persona con } \\
\text { discapacidad pueda ayudar a los } \\
\text { demás }\end{array}$ & & & & & & \\
\hline $\begin{array}{l}\text { Las personas con discapacidad no } \\
\text { resultan atractivas para otras perso- } \\
\text { nas }\end{array}$ & & & & & & \\
\hline $\begin{array}{l}\text { ¿Has tenido interacción con alguna } \\
\text { persona con discapacidad? }\end{array}$ & \multicolumn{3}{|l|}{$\mathrm{Si}$} & \multicolumn{3}{|l|}{ No } \\
\hline Sexo & \multicolumn{3}{|l|}{ Varón } & \multicolumn{3}{|l|}{ Mujer } \\
\hline \multicolumn{7}{|l|}{ Edad } \\
\hline \multicolumn{7}{|l|}{ Tituación } \\
\hline Curso & & & & & & \\
\hline
\end{tabular}

